Journal for ImmunoTherapy of Cancer

\title{
Circulating cytokines associated with clinical response to systemic therapy in metastatic renal cell carcinoma
}

To cite: Chehrazi-Raffle A, Meza L, Alcantara M, et al. Circulating cytokines associated with clinical response to systemic therapy in metastatic renal cell carcinoma. Journal for ImmunoTherapy of Cancer 2021;9:e002009. doi:10.1136/ jitc-2020-002009

- Additional material is published online only. To view, please visit the journal online (http://dx.doi.org/10.1136/jitc2020-002009).

AC-R, LM and MA contributed equally.

Accepted 29 December 2020

D Check for updates

(C) Author(s) (or their employer(s)) 2021. Re-use permitted under CC BY-NC. No commercial re-use. See rights and permissions. Published by BMJ.

For numbered affiliations see end of article.

Correspondence to

Dr Sumanta Pal; spal@coh.org

\section{ABSTRACT}

Background Circulating cytokines and angiogenic factors have been associated with clinical outcomes in patients with metastatic renal cell carcinoma (RCC) receiving systemic therapy. However, none have yet examined cytokine concentrations in parallel cohorts receiving either immunotherapy or targeted therapy.

Methods In this prospective correlative study, we enrolled 56 patients who were planned for treatment with either a vascular endothelial growth factor-tyrosine kinase inhibitor (VEGF-TKI) or immune checkpoint inhibitor (ICI). Eligibility requirements permitted any RCC histologic subtype, International Metastatic Renal Cell Carcinoma risk classification, and line of therapy. Immunologic profile was assessed at baseline and after 1 month on treatment using a Human Cytokine 30-plex protein assay (Invitrogen). Clinical benefit was defined as complete response, partial response, or stable disease $\geq 6$ months per RECIST (Response Evaluation Criteria in Solid Tumors) V.1.1 criteria.

Results Clinical benefit was similar between VEGFTKI and ICI arms (65\% vs $54 \%$ ). Patients with clinical benefit from VEGF-TKIs had lower pretreatment levels of interleukin-6 (IL-6) ( $p=0.02)$, IL-1RA ( $p=0.03)$, and granulocyte colony-stimulating factor (CSF) $(p=0.02)$. At 1 month, patients with clinical benefit from ICls had higher levels of interferon- $\gamma(\mathrm{IFN}-\gamma)(p=0.04)$ and $\mathrm{LL}-12$ $(p=0.03)$. Among patients on VEGF-TKIs, those with clinical benefit had lower 1 month IL-13 $(p=0.02)$ and granulocyte macrophage CSF $(p=0.01)$ as well as higher 1 month VEGF $(p=0.04)$ compared with patients with no clinical benefit. Conclusion For patients receiving VEGF-TKI or ICI therapy, distinct plasma cytokines were associated with clinical benefit. Our findings support additional investigation into plasma cytokines as biomarkers in metastatic RCC.

\section{INTRODUCTION}

The landscape of systemic therapy for metastatic renal cell carcinoma (RCC) has undergone considerable change in recent years. At present, immune checkpoint inhibitors (ICIs), vascular endothelial growth factortyrosine kinase inhibitors (VEGF-TKIs), and combination therapy with both an ICI and a VEGF-TKI are approved by the US Food and Drug Administration (FDA). ${ }^{1}$ With numerous treatment options and no firm consensus regarding their optimal sequence, biomarkers to select patients who will benefit from systemic therapies are urgently needed.

RCC is largely driven by the loss of von Hippel-Lindau protein, which enables uncontrolled activation of hypoxia-inducible factor- $\alpha$ and subsequent upregulation of numerous proangiogenic cytokines. ${ }^{23}$ Twin studies have established that variations in circulating cytokine concentrations are primarily driven by non-heritable influences, which suggests they may be used as biomarkers for individualizing treatment of RCC among other acquired disease states. ${ }^{45}$ As such, peripheral cytokine assays have garnered considerable interest as potential functional biomarkers that may aid in individualizing treatment selection and avoiding potentially harmful adverse events.

Several groups have explored cytokine concentrations as potential biomarkers for patients with RCG on VEGF-TKIs. Bilen et $a t^{6}$ identified a panel of seven cytokines that were negatively associated with clinical benefit from first-line sunitinib, including interleukin-8 (IL-8), IL-9, and tumor necrosis factor- $\alpha$ (TNF- $\alpha)$. Tran and colleagues retrospectively noted negative associations between progression-free survival from frontline pazopanib and pretreatment IL-6, IL-8, VEGF, osteopontin, hepatocyte growth factor (HGF), and E-selectin. ${ }^{7}$ Zizzari $e t a l^{8}$ demonstrated that interferon- $\gamma$ (IFN- $\gamma)$, soluble programmed death ligand 1 (PD-L1), and soluble cytotoxic T-lymphocyte antigen 4 (CTLA-4) were inversely correlated with a clinical benefit from sunitinib or pazopanib. Within our institution, we prospectively replicated negative associations between clinical benefit from pazopanib and levels of HGF, VEGF, IL-6, IL-8, and soluble IL-2R. ${ }^{9}$

Similarly, investigators have examined outcomes using cytokines as biomarkers for 
patients with RCC on ICIs. Transcriptional analysis established that nivolumab induces elevated peripheral levels of IFN- $\gamma$, CXCL-9, and CXCL-10. ${ }^{10}$ Additionally, baseline IL-8 has been implicated as a negative prognosticator for ICI's effectiveness across several tumor types, including RCC and urothelial carcinoma. ${ }^{11}{ }^{12}$ More recently, machine-learning techniques identified an eight-cytokine composite immune signature that was prognostic for overall survival for patients with RCC on nivolumab. ${ }^{13}$

Lacking in the existing literature is parallel assessment of cytokines in patients receiving VEGF-TKIs or ICIs. To address this, we conducted a prospective trial to determine if various pretreatment and on-treatment cytokine levels in peripheral blood predict clinical outcomes for patients with metastatic RCC receiving ICI or VEGF-TKI therapies.

\section{METHODS}

\section{Patient selection and sample acquisition}

Patients diagnosed with metastatic RCC by standard criteria (American Joint Committee on Cancer Seventh Edition) were identified at a single institution. ${ }^{14}$ All subjects were planned for treatment with either a commercially available ICI or VEGF-TKI therapy approved for metastatic RCC by the US FDA. Prior therapeutic failure of other ICI or VEGF-TKI agents did not preclude patients from participating in this study. Enrollment was open to patients across all RCC histological subtypes, International Metastatic Renal Cell Carcinoma (IMDC) risk classification, and lines of therapy (online supplemental table 1).

The protocol was approved by the institutional scientific review committee, data safety monitoring board, and the institutional review board at the City of Hope Comprehensive Cancer Center. The study conformed with the amended Declaration of Helsinki and the International Conference on Harmonization Guidelines.

\section{Cytokine analysis}

Peripheral blood samples were obtained from each patient prior to and at 1 month subsequent to the initiation of systemic therapy with either a VEGF-TKI or ICI. Plasma samples were separated using centrifugation in cell preparation tubes (BD Biosciences, San Jose, California, USA) at $1800 \times \mathrm{g}$ for $20 \mathrm{~min}$ at room temperature, then frozen at $-80^{\circ} \mathrm{C}$ for subsequent batched analysis. A panel of 30 plasma cytokines (IL-1RA, IL-1b, IL-2, IL-2R, IL-4, IL-5, IL-6, IL-7, IL-8, IL-10, IL-12, IL-13, IL-15, IL-17, Eotaxin, EGF, FGF, G-CSF, GM-CSF, IFN- $\alpha$, IGN- $\gamma$, CXCL9, CXCL10, CCL2, CCL3, CCL4, RANTES, TNF- $\alpha$, and VEGF) was quantified for each plasma sample using the Human Cytokine Invitrogen 30-plex protein assay on the Luminex FLEXMAP 3D System (Fisher-Scientific, Waltham, Massachusetts, USA). In brief, antibodycoated beads were incubated with plasma sample, incubation buffer, and assay diluent. Detector antibody was added and incubated followed by the addition of diluted
streptavidin-RPE. After washing, the plate was read in the Luminex detection system to quantify cytokine abundance.

\section{Clinical data analysis}

Patients were treated with either an ICI or VEGF-TKI until disease progression per Response Evaluation Criteria in Solid Tumors (RECIST) V.1.1 criteria or discontinuation due to adverse events, death, or subject/investigator decision. Clinical benefit (CB) was classified as a (CR), partial response $(\mathrm{PR})$, or stable disease $(\mathrm{SD})$ in excess of 6 months per clinician's evaluation of CT of the chest, abdomen, and pelvis. Individuals experiencing progressive disease (PD) on therapy or who achieved SD of less than 6 months were classified as experiencing no clinical benefit (NCB).

To arrive at relative change from baseline, ratio of 1 month to baseline cytokine values were calculated. Median and IQR were used to summarize cytokine data at baseline as well as relative change at 1 month. The Wilcoxon test was used to assess statistical significance in differences of cytokine values between $\mathrm{CB}$ and $\mathrm{NCB}$ patients within VEGF-TKI and ICI cohorts. The $\chi^{2}$ test was used to determine statistical significance in comparison of high versus low cytokine values between $\mathrm{CB}$ and $\mathrm{NCB}$ patients.

The significance threshold for type I error was set at 0.05 . Given the exploratory nature of the analysis, no formal correction for multiple testing was applied. SAS V.9.4 was used to perform statistical analyses and generate graphs.

\section{RESULTS}

\section{Patient characteristics}

We prospectively analyzed 56 patients who were treated at City of Hope Comprehensive Cancer Center between 2016 and 2019 (table 1). Of these, $40(73 \%)$ patients were male and $16(27 \%)$ were female, with a median age of 67 (range 32-85). Median lines of therapy received was 2 (range 1-6). The most common ICIs rendered were nivolumab monotherapy (45\%) and nivolumab plus ipilimumab (14\%), and the most frequent VEGF-TKIs given were cabozantinib (18\%), sunitinib (13\%), lenvatinib/ everolimus (9\%) and axitinib (2\%).

Among those eligible for evaluation, 7 patients (13\%) achieved a PR and 25 patients $(45 \%)$ achieved SD as a best response. Seventeen patients $(30 \%)$ experienced PD and seven (13\%) discontinued treatment due to toxicity. $\mathrm{CB}$ was similar in patients receiving VEGF-TKI and ICI (65\% vs $54 \%$, respectively). Treatment-related toxicities were reported in 36 evaluable patients (64\%). The most common reported toxicities were fatigue $(21 \%)$ with ICIs and hand-foot syndrome (17\%) with VEGF-TKIs.

\section{Biomarker results}

Cytokine data were available at baseline and at 1 month for 56 and 47 patients, respectively. Patients were stratified 
Table 1 Summary of patient baseline characteristics and clinical outcomes

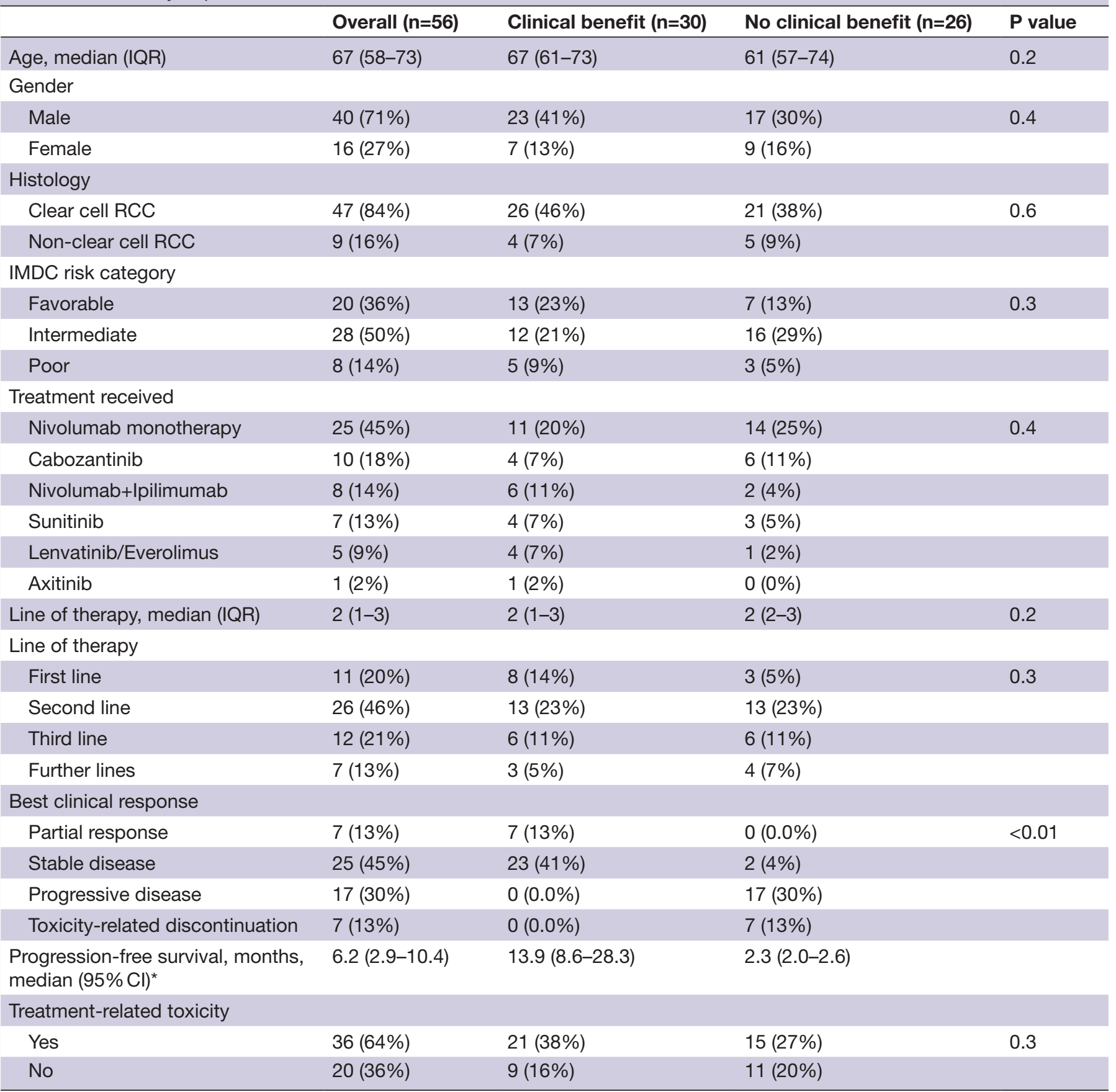

*Kaplan-Meier method.

IMDC, International Metastatic Renal Cell Carcinoma Consortium; RCC, renal cell carcinoma.

based on clinical benefit status: $\mathrm{CB}(\mathrm{n}=30)$ or NCB $(\mathrm{n}=26)$. Patients were further grouped based on therapy received, resulting in four analyzable groups: CB-VEGF-TKI $(n=13)$, CB-ICI $(n=17)$, NCB-VEGF-TKI $(n=10)$ and NCB-ICI $(\mathrm{n}=16)$.

Patients with CB from VEGF-TKIs had lower pretreatment median levels of IL-6 (8.4 vs $13.5 \mathrm{pg} / \mathrm{mL}, \mathrm{p}=0.02)$, IL-1RA (178 vs $248 \mathrm{pg} / \mathrm{mL}, \mathrm{p}=0.03$ ), and G-CSF (23.9 vs $38.3 \mathrm{pg} / \mathrm{mL}, \mathrm{p}=0.02)$ compared with patients with $\mathrm{NCB}$ (table 2A and figure 1). No significant pretreatment cytokine differences were seen among $\mathrm{CB}$ and $\mathrm{NCB}$ patients on ICIs.

Additionally, we assessed the cytokine concentration change from baseline to 1 month on therapy. To normalize values, we then converted these relative changes into ratio form. Patients who derived CB from ICIs had higher relative increases in IFN- $\gamma(2.04$ vs $0.91, \mathrm{p}=0.04)$ and IL-12 (1.51 vs $1.01, \mathrm{p}=0.03)$. For patients on VEGF-TKIs, those with $\mathrm{CB}$ had lower relative IL-13 (0.56 vs $0.93, \mathrm{p}=0.02)$ and GM-CSF (0.94 vs $1.24, \mathrm{p}=0.01$ ), and higher relative VEGF (1.69 vs 
Table 2 Associations of (A) pretreatment and (B) ontreatment cytokine levels with clinical benefit from systemic therapy

\begin{tabular}{|c|c|c|c|c|}
\hline \multicolumn{2}{|c|}{ (A) Pretreatment $n$} & CB & NCB & $P$ value \\
\hline \multicolumn{5}{|c|}{ G-CSF (VEGF-TKI) } \\
\hline High & 11 & $5(38.5 \%)$ & $6(66.7 \%)$ & 0.02 \\
\hline Low & 11 & $8(61.5 \%)$ & $3(33.3 \%)$ & \\
\hline \multicolumn{5}{|c|}{ IL-1RA (VEGF-TKI) } \\
\hline High & 9 & $4(30.8 \%)$ & $5(55.6 \%)$ & 0.03 \\
\hline Low & 13 & $9(69.2 \%)$ & $4(44.4 \%)$ & \\
\hline \multicolumn{5}{|c|}{ IL-6 (VEGF-TKI) } \\
\hline High & 11 & $2(15.4 \%)$ & $7(77.8 \%)$ & 0.02 \\
\hline Low & 11 & $11(84.6 \%)$ & $2(22.2 \%)$ & \\
\hline $\begin{array}{l}\text { (B) On- } \\
\text { treatment }\end{array}$ & $\mathbf{n}$ & CB & NCB & $P$ value \\
\hline \multicolumn{5}{|c|}{ GM-CSF (VEGF-TKI) } \\
\hline High & 10 & $3(25.0 \%)$ & 7 (77.8\%) & 0.01 \\
\hline Low & 11 & $9(75.0 \%)$ & $2(22.2 \%)$ & \\
\hline \multicolumn{5}{|l|}{$\mathrm{IFN}-\gamma(\mathrm{ICl})$} \\
\hline High & 10 & $9(64.3 \%)$ & $1(9.1 \%)$ & 0.04 \\
\hline Low & 15 & $5(35.7 \%)$ & $10(90.9 \%)$ & \\
\hline \multicolumn{5}{|l|}{$\mathrm{IL}-12(\mathrm{ICl})$} \\
\hline High & 15 & $10(71.4 \%)$ & $5(45.4 \%)$ & 0.03 \\
\hline Low & 10 & $4(28.6 \%)$ & $6(54.6 \%)$ & \\
\hline \multicolumn{5}{|c|}{ IL-13 (VEGF-TKI) } \\
\hline High & 12 & $4(33.3 \%)$ & $8(88.9 \%)$ & 0.02 \\
\hline Low & 9 & $8(66.7 \%)$ & $1(11.1 \%)$ & \\
\hline \multicolumn{5}{|c|}{ VEGF (VEGF-TKI) } \\
\hline High & 15 & $11(91.7 \%)$ & $4(44.4 \%)$ & 0.04 \\
\hline Low & 6 & $1(8.3 \%)$ & $5(55.6 \%)$ & \\
\hline
\end{tabular}

The $\chi^{2}$ test was used to determine statistical significance in comparison of high versus low cytokine values between $\mathrm{CB}$ and NCB patients.

$\mathrm{CB}$, clinical benefit ; G-CSF, granulocyte colony-stimulating factor; GM-CSF, granulocyte macrophage colony-stimulating factor; $\mathrm{ICl}$, immune checkpoint inhibitor; IFN- $\gamma$, interferon- $\gamma$; IL, interleukin; NCB, no clinical benefit; VEGF-TKI, vascular endothelial growth factor-tyrosine kinase inhibitor.

$0.81, \mathrm{p}=0.04$ ) as compared with those with $\mathrm{NCB}$ (table 2B and figure 2).

\section{DISCUSSION}

This is the first prospective study involving analyses of baseline and on-treatment cytokine concentrations on ICI or VEGF-TKI therapy. We found that pretreatment levels of IL-1RA, IL-6, and G-CSF as well as on-treatment levels of IL-12, IL-13, IFN- $\gamma$, GM-CSF, and VEGF were associated with $\mathrm{CB}$ from systemic therapy.

Among cytokines analyzed pretreatment, we observed a negative association between IL-6 concentrations and
CB in patients who received a VEGF-TKI. Previous studies have also observed an inverse relationship between $\mathrm{CB}$ and levels of IL-6 in RCC. ${ }^{715} 16$ One of the key biological pathways governing this phenomenon is the IL-6/ JAK/STAT3 axis, which potentiates tumor proliferation and cellular metabolism on upregulation. ${ }^{17} 18$ Extrinsic to the tumor milieu, IL-6 promotes tumor survival by recruiting mesenchymal stem cells and myeloid-derived suppressor cells (MDSCs). Mesenchymal stem cells are non-immune cells that, when engaged with IL-6, can suppress tumor-infiltrating CD8+ T cells and B cells in the tumor microenvironment and thus mitigate tumor immunity. ${ }^{19-21}$ Similarly, MDSCs, which require IL-6 for activation, have been shown to suppress $\mathrm{T}$ cell response and facilitate cancer persistence via the JAK/STAT3 signaling pathway. $^{22-24}$

Another tumorigenic by-product of IL-6 is upregulation of angiogenesis. This occurs both within the tumor by autocrine and/or paracrine signaling and by tumorextrinsic positive-feedback amplification of STAT3 transcription factor, which increases expression of proangiogenic VEGF and HIF $1 \alpha{ }^{25-27}$ Elevated levels of pretreatment IL-6 may therefore serve as a harbinger of a highly proliferative and angiogenic state that will be less effectively abrogated by treatment with a VEGF-TKI.

Another important negative association evident in our VEGF-TKI cohort was between CB and pretreatment IL-1RA. IL-1 is a potent proinflammatory cytokine that promotes tumorigenesis through several pathways, including angiogenesis, endothelial cell activation, lymphoid cell polarization, and recruitment of tumorassociated macrophages and MDSCs. ${ }^{28}$ By extension, IL-1RA has long been thought to antagonize carcinogenesis via inhibition of IL- $1 \alpha$ and IL-1B. ${ }^{29}{ }^{30}$ Intriguingly, emerging data of IL-1RA in advanced diseases are beginning to unravel its role in caspase- 8 and caspase- 9 inhibition, which would evade apoptosis and facilitate oncogenesis. ${ }^{31}{ }^{32}$ Therefore, elevated IL-1RA concentrations may in fact signal a highly proliferative RCC that is less responsive to VEGF-TKI therapy.

Similarly, baseline concentrations of granulocyte colonystimulating factor (G-CSF) and on-treatment concentrations of granulocyte macrophage colony-stimulating factor (GM-CSF) were inversely associated with $\mathrm{CB}$ from VEGF-TKI. In preclinical models, RCC cell lines produce G-CSF without any counter-regulatory dependence on exogenous G-CSF. ${ }^{33}$ It has also been shown that G-CSF and GM-CSF can increase the motility and invasiveness of tumor cells as well as enhance the metastatic process. ${ }^{34} 35$ Furthermore, higher levels of intratumoral GM-CSF have been associated with the presence of lymph node metastasis, high tumor, node, metastases (TNM) stage, Fuhrman grade and the presence of tumor necrosis. ${ }^{36}$ In this context, RCC with higher concentrations of G-CSF and GM-CSF may be indicative of an imbalanced immunoregulation that fares worse with VEGF-TKI therapy.

Our most robust associations in patients who derived CB from ICIs were seen from on-treatment increases of 


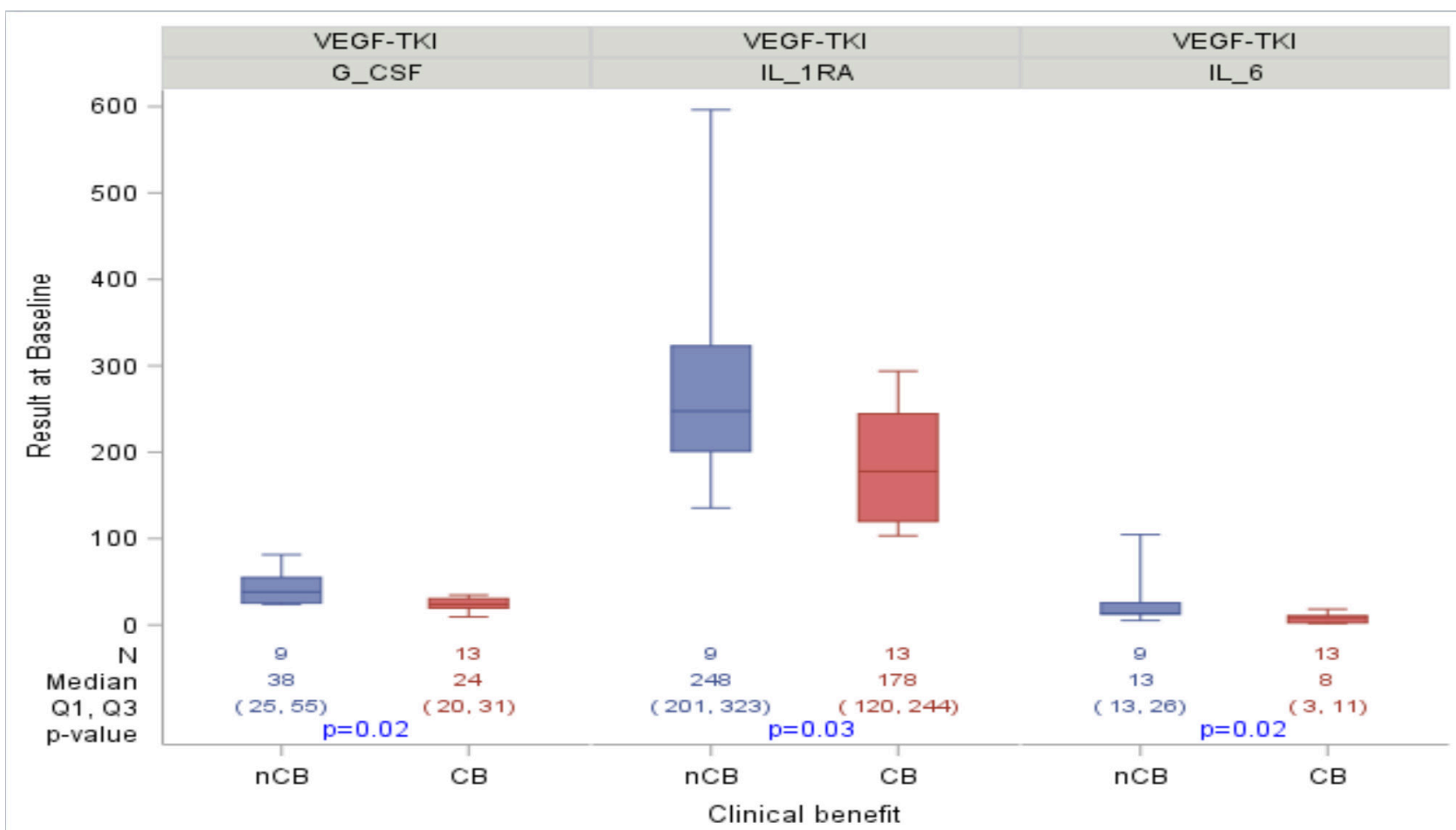

Figure 1 Associations between clinical benefit from vascular endothelial growth factor-tyrosine kinase inhibitor (VEGF-TKI) therapy and pretreatment interleukin-6 (IL-6), granulocyte colony-stimulating factor (G-CSF), and IL-1RA. Box contains values $\mathrm{q} 1$, median and q2. Whiskers expand out to 10th and 90th percentiles. P values are from the Wilcoxon rank-sum test. CB, clinical benefit; NCB, no clinical benefit.

IL-12 ( $\mathrm{p}=0.03)$ and IFN- $\gamma(\mathrm{p}=0.04)$. IL-12 is a key proinflammatory and anti-angiogenic cytokine that antagonizes tumor growth via stimulation of STAT4, which then subsequently upregulates IFN- $\gamma^{37}$ Once activated, IFN- $\gamma$ can facilitate a cell-mediated adaptive immune response

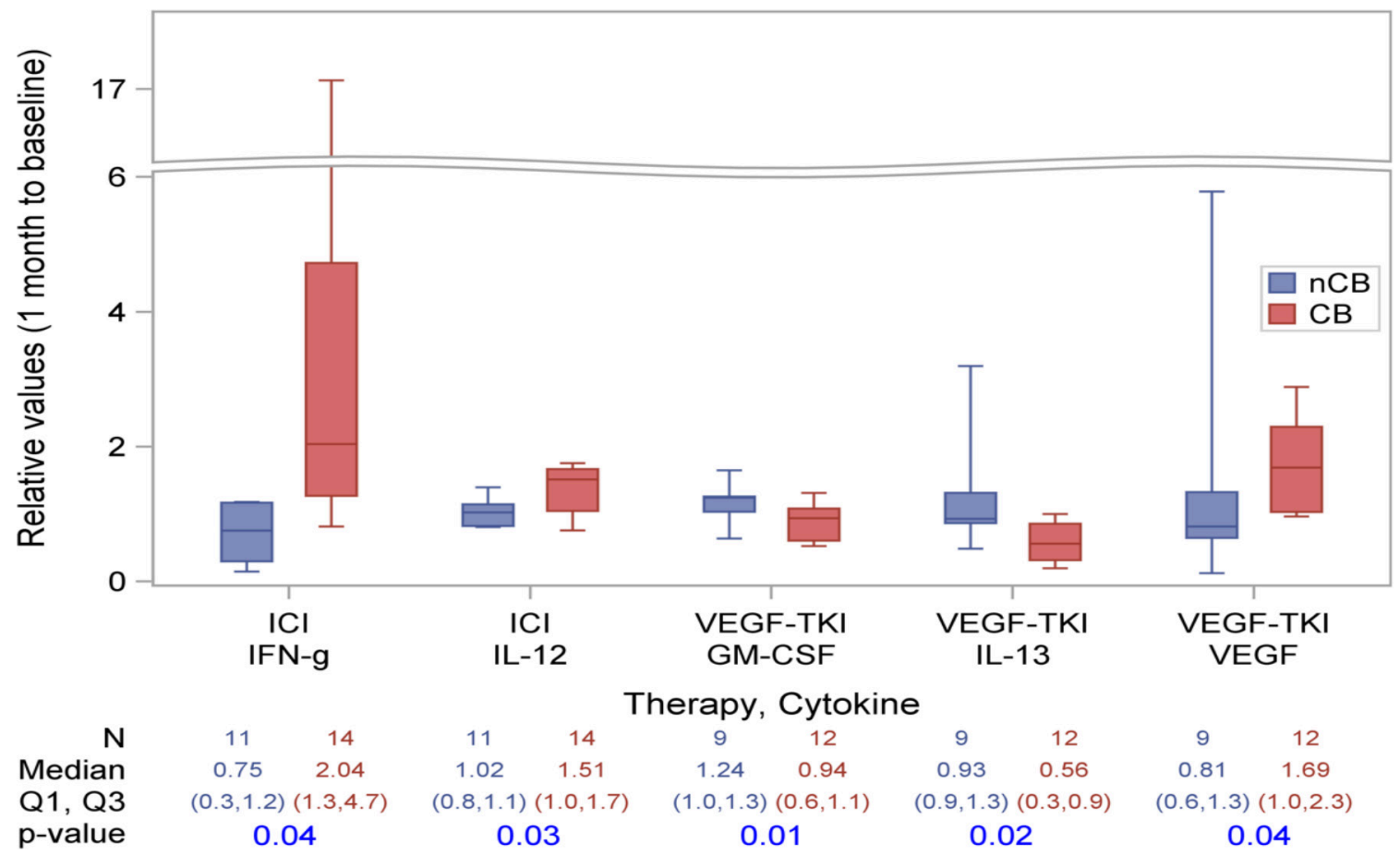

Figure 2 Associations between on-treatment cytokine changes and clinical benefit either vascular endothelial growth factortyrosine kinase inhibitor (VEGF-TKI) or immune checkpoint inhibitor (ICI) therapy. Box contains values q1, median and q2. Whiskers expand out to 10th and 90th percentiles. P values are from the Wilcoxon rank-sum test. CB, clinical benefit; GM-CSF, granulocyte macrophage colony-stimulating factor; IFN, interferon; IL, interleukin; NCB, no clinical benefit. 
through the IFN- $\gamma / \mathrm{JAK} / \mathrm{STAT} 1$ pathway promoting T-cell-mediated antitumor immune responses. ${ }^{38}$ The importance of IL-12 in cancer has been demonstrated in murine models where IL-12 deficiency was associated with a higher frequency of spontaneous tumors and enhanced tumor growth. ${ }^{39} 40$ In humans, IL-12 polymorphisms have been shown to increase the susceptibility of glioblastoma development. $^{41}$

With respect to ICIs, several preclinical models have demonstrated that IL-12 and IFN- $\gamma$ play a pivotal role in facilitating an anticancer response to PD-1/PD-L1 inhibitors. Treatment with ICIs is accompanied by elevated plasma concentrations of IFN- $\gamma$ as well as an influx in IFN- $\gamma$-producing $\mathrm{T}$ cells, both in peripheral blood and tumor tissues. ${ }^{42}$ Similarly, intratumoral IL-12 was shown to reverse resistance to anti-PD-1 therapy in T-cell-rich tumors. ${ }^{43}$ More recently, anti-PD-1 treatment was associated with an increase in intratumoral CD8+ T cells and dendritic cell (DC)-like cells that increased IFN- $\gamma$ and IL-12, respectively. ${ }^{44}$ Taken together, increasing IL-12 and IFN- $\gamma$ may reflect a clinically efficacious anticancer immune response from ICIs.

We also detected that a greater decrease in on-treatment IL-13 was associated with CB from VEGF-TKI. IL-13 is a pleiotropic cytokine that is overexpressed in a variety of solid tumors. ${ }^{45}{ }^{46}$ One of the downstream targets of IL-13 implicated in cancer progression is STAT6, which promotes apoptotic resistance by downregulating tumor immunosurveillance. ${ }^{47-49}$ However, IL-13 also activates the 15-lipoxygenase-1 pathway, which has been shown to induce apoptosis and inhibit proliferation of cancer cells. ${ }^{49-51}$

Not surprisingly, previous research on the role of IL-13 in malignancies has generated conflicting evidence. Although several studies have facilitated a protumor effect, ${ }^{52-54}$ others have elucidated a protective environment. ${ }^{556}$ With respect to RCC, IL-13 has been associated with shorter recurrence-free survival and overall survival in post-nephrectomy patients. ${ }^{57}$ Our findings corroborate a predominance of pro-tumorigenesis, supporting IL-13 as a promising negative prognostic biomarker in RCC.

Lastly, we observed significantly greater increases in on-treatment VEGF among patients who benefited from VEGF-TKI. Upregulation of plasma VEGF is a well-documented feedback mechanism of patients on VEGF-TKI therapy, although the mechanism behind this observation remains unclear. ${ }^{58-60}$ Initial enthusiasm for its application as a predictive biomarker for VEGF-TKI therapy was tempered by inconsistent validation. However, subsequent studies have yielded encouraging results by grouping VEGF along with other pro-angiogenic cytokines as part of a broader immune signature. ${ }^{7135861}$ The development of an oncogenic immune signature that accounts for the dynamic interplay of cytokines is a promising future direction that warrants further investigation.

There are several limitations to the present study. We included a small number of patients and did not include a healthy control arm. Subjects included in this study were sourced from a single hospital and may not be representative of the general population. The heterogeneity of therapy line, and thus, previous exposure to VEGF-TKI and/or ICI therapy, posed an additional limitation which is not controlled for within this study. Moreover, it should be noted that the inclusion of five patients on lenvatinib plus everolimus, a mechanistic target of rapamycin (mTOR) inhibitor, likely introduces immunomodulatory effects that are extrinsic to the VEGF-TKI pathways shared by the other regimens within this cohort. In addition, lifestyle-related variables, which may affect the levels of these cytokines, were not accounted for in the present study. ${ }^{62}$

\section{CONCLUSIONS}

In the present study, we detected associations between peripheral cytokine concentrations and response to systemic therapy in patients with metastatic RCC. Pretreatment IL-6, IL-1RA, and G-CSF-as well as on-treatment IL-12, IL-13, IFN- $\gamma$, GM-CSF, and VEGF-were significantly associated with CB from either VEGF-TKIs or ICIs. Although exploratory, these data support the further in-depth study of plasma cytokines as biomarkers for immune phenotype stratification in order to provide more individualized treatment for metastatic RCC.

\section{Author affiliations}

${ }^{1}$ Department of Medical Oncology and Therapeutics Research, City of Hope Comprehensive Cancer Center, Duarte, California, USA

${ }^{2}$ Department of Immuno-Oncology, City of Hope Comprehensive Cancer Center, Duarte, California, USA

${ }^{3}$ Department of Internal Medicine, Yale School of Medicine, New Haven, Connecticut, USA

${ }^{4}$ Department of Medical Oncology, Cettro Cancer Center, Brasilia, Brazil ${ }^{5}$ Jacobs School of Medicine and Biomedical Sciences, Buffalo, New York, Brazil ${ }^{6}$ Department of Computational and Quantitative Medicine, City of Hope Comprehensive Cancer Center, Duarte, California, USA

Twitter Alexander Chehrazi-Raffle @arafflemd and Nazli Dizman @NazliDizman

Contributors Study concept and design: AC-R, LM, ND, PB, JH, and SP. Acquisition analysis, or interpretation of data: All authors. Drafting of the manuscript: AC-R, LM, and SP. Critical revision of the manuscript for important intellectual content: All authors. Statistical analysis: AC-R, EK, MA, MK, and SP. Study supervision: SP.

Funding Pfizer Inc. and Bristol-Myers Squibb have sponsored this study.

Competing interests SP reports consulting roles in Genentech, Aveo, Eisai, Roche, Pfizer, Novartis, Exelixis, Ipsen, BMS, and Astellas. ACR, LM, MA, ND, PB, NS, JH, NR, EK, and MK declare no conflicts of interest.

Patient consent for publication Not required.

Ethics approval Sample collection and usage was approved by the Ethics Review Committees of City of Hope Comprehensive Cancer Center and in accordance with the Declaration of Helsinki.

Provenance and peer review Not commissioned; externally peer reviewed.

Data availability statement Data are available upon reasonable request. Our data consists of deidentified participant information that was collected as part of an IRB-approved protocol. Cytokine and clinical data are available upon request to $\mathrm{Dr}$ Marcin Kortylewski at mkortylewski@coh.org.

Open access This is an open access article distributed in accordance with the Creative Commons Attribution Non Commercial (CC BY-NC 4.0) license, which permits others to distribute, remix, adapt, build upon this work non-commercially, 
and license their derivative works on different terms, provided the original work is properly cited, appropriate credit is given, any changes made indicated, and the use is non-commercial. See http://creativecommons.org/licenses/by-nc/4.0/.

\section{ORCID iDs}

Nazli Dizman http://orcid.org/0000-0002-3907-8513

Sumanta Pal http://orcid.org/0000-0002-1712-0848

\section{REFERENCES}

1 National Cancer Institute. Drugs Approved for kidney (renal cell) cancer, 2011. Available: https://www.cancer.gov/about-cancer/ treatment/drugs/kidney

2 Kaelin WG. Von Hippel-Lindau disease. Annu Rev Pathol 2007:2:145-73.

3 Majmundar AJ, Wong WJ, Simon MC. Hypoxia-Inducible factors and the response to hypoxic stress. Mol Cell 2010;40:294-309.

4 de Craen AJM, Posthuma D, Remarque EJ, et al. Heritability estimates of innate immunity: an extended twin study. Genes Immun 2005;6:167-70

5 Brodin P, Jojic V, Gao T, et al. Variation in the human immune system is largely driven by non-heritable influences. Cell 2015;160:37-47.

6 Bilen MA, Zurita AJ, Ilias-Khan NA, et al. Hypertension and circulating cytokines and angiogenic factors in patients with advanced Non-Clear cell renal cell carcinoma treated with sunitinib: results from a phase II trial. Oncologist 2015;20:1140-8.

7 Tran HT, Liu Y, Zurita AJ, et al. Prognostic or predictive plasma cytokines and angiogenic factors for patients treated with pazopanib for metastatic renal-cell cancer: a retrospective analysis of phase 2 and phase 3 trials. Lancet Oncol 2012;13:827-37.

8 Zizzari IG, Napoletano C, Di Filippo A, et al. Exploratory pilot study of circulating biomarkers in metastatic renal cell carcinoma. Cancers 2020;12:2620.

9 Pal SK, Hossain DMS, Zhang Q, et al. Pazopanib as third line therapy for metastatic renal cell carcinoma: clinical efficacy and temporal analysis of cytokine profile. J Urol 2015;193:1114-21.

10 Choueiri TK, Fishman MN, Escudier B, et al. Immunomodulatory activity of nivolumab in metastatic renal cell carcinoma. Clin Cancer Res 2016;22:5461-71.

11 Schalper KA, Carleton M, Zhou M, et al. Elevated serum interleukin-8 is associated with enhanced intratumor neutrophils and reduced clinical benefit of immune-checkpoint inhibitors. Nat Med 2020;26:688-92.

12 Bakouny Z, Choueiri TK. II-8 and cancer prognosis on immunotherapy. Nat Med 2020;26:650-1.

13 Wang R, Zheng J, Shao X, et al. Development of a prognostic composite cytokine signature based on the correlation with nivolumab clearance: translational PK/PD analysis in patients with renal cell carcinoma. J Immunother Cancer 2019;7:348.

14 Amin MB, Edge S, Greene F, eds. AJCC cancer staging manual. 8th ed. New York: Springer International Publishing, 2017.

15 Negrier S, Perol D, Menetrier-Caux C, et al. Interleukin-6, interleukin-10, and vascular endothelial growth factor in metastatic renal cell carcinoma: prognostic value of interleukin-6--from the Groupe Francais d'Immunotherapie. J Clin Oncol 2004;22:2371-8.

16 Zurita AJ, Gagnon RC, Liu Y, et al. Integrating cytokines and angiogenic factors and tumour bulk with selected clinical criteria improves determination of prognosis in advanced renal cell carcinoma. Br J Cancer 2017;117:478-84.

17 Ishibashi K, Koguchi T, Matsuoka K, et al. Interleukin-6 induces drug resistance in renal cell carcinoma. Fukushima J Med Sci 2018;64:103-10.

18 Chang Q, Bournazou E, Sansone P, et al. The IL-6/JAK/Stat3 feed-forward loop drives tumorigenesis and metastasis. Neoplasia 2013;15:848-IN45

19 Ling W, Zhang J, Yuan Z, et al. Mesenchymal stem cells use IDO to regulate immunity in tumor microenvironment. Cancer Res 2014;74:1576-87.

20 Liotta F, Querci V, Mannelli G, et al. Mesenchymal stem cells are enriched in head neck squamous cell carcinoma, correlates with tumour size and inhibit T-cell proliferation. Br J Cancer 2015;112:745-54.

21 Sun Z, Wang S, Zhao RC. The roles of mesenchymal stem cells in tumor inflammatory microenvironment. J Hematol Oncol 2014:7:14.

$22 \mathrm{Yu} \mathrm{H}$, Pardoll D, Jove R. Stats in cancer inflammation and immunity: a leading role for STAT3. Nat Rev Cancer 2009;9:798-809.

23 Huang B, Pan P-Y, Li Q, et al. Gr-1+CD115+ immature myeloid suppressor cells mediate the development of tumor-induced $\mathrm{T}$ regulatory cells and T-cell anergy in tumor-bearing host. Cancer Res 2006:66:1123-31.

24 Veglia F, Perego M, Gabrilovich D. Myeloid-Derived suppressor cells coming of age. Nat Immunol 2018;19:108-19.

25 Lederle W, Depner S, Schnur S, et al. II-6 promotes malignant growth of skin SCCs by regulating a network of autocrine and paracrine cytokines. Int J Cancer 2011;128:2803-14.

$26 \mathrm{Yu} \mathrm{H}$, Kortylewski M, Pardoll D. Crosstalk between cancer and immune cells: role of STAT3 in the tumour microenvironment. Nat Rev Immunol 2007;7:41-51.

27 Kujawski M, Kortylewski M, Lee $\mathrm{H}$, et al. Stat3 mediates myeloid cell-dependent tumor angiogenesis in mice. J Clin Invest 2008;118:3367-77.

28 Mantovani A, Barajon I, Garlanda C. II-1 and IL-1 regulatory pathways in cancer progression and therapy. Immunol Rev 2018;281:57-61

29 Chirivi RG, Garofalo A, Padura IM, et al. Interleukin 1 receptor antagonist inhibits the augmentation of metastasis induced by interleukin 1 or lipopolysaccharide in a human melanoma/nude mouse system. Cancer Res 1993;53:5051-4.

30 McKenzie RC, Oran A, Dinarello CA, et al. Interleukin-1 receptor antagonist inhibits subcutaneous B16 melanoma growth in vivo. Anticancer Res 1996;16:437-41.

31 Spinello A, Vecile E, Abbate A, et al. How can interleukin-1 receptor antagonist modulate distinct cell death pathways? J Chem Inf Model 2019;59:351-9.

32 Vecile E, Dobrina A, Salloum FN, et al. Intracellular function of interleukin-1 receptor antagonist in ischemic cardiomyocytes. PLoS One 2013;8:e53265.

33 Gerharz CD, Reinecke P, Schneider EM, et al. Secretion of GM-CSF and M-CSF by human renal cell carcinomas of different histologic types. Urology 2001;58:821-7.

34 Nicoletti G, Lollini PL, Bagnara GP, et al. Are colony-stimulating factor-producing cells facilitated in the metastatic process? Anticancer Res 1987;7:695-700.

35 Young MR, Halpin J, Hussain R, et al. Inhibition of tumor production of granulocyte-macrophage colony-stimulating factor by 1 alpha, 25-dihydroxyvitamin D3 reduces tumor motility and metastasis. Invasion Metastasis 1993;13:169-77.

36 Chang Y, Xu L, Zhou L, et al. Granulocyte macrophage colonystimulating factor predicts postoperative recurrence of clear-cell renal cell carcinoma. Oncotarget 2016;7:24527-36.

37 Yan J, Smyth MJ, Teng MWL. Interleukin (IL)-12 and IL-23 and Their Conflicting Roles in Cancer. Cold Spring Harb Perspect Biol 2018;10. doi:10.1101/cshperspect.a028530. [Epub ahead of print: 0207 2018]

38 Zaidi MR, Merlino G. The two faces of interferon- $\gamma$ in cancer. Clin Cancer Res 2011;17:6118-24.

39 Tugues S, Burkhard SH, Ohs I, et al. New insights into IL-12mediated tumor suppression. Cell Death Differ 2015;22:237-46.

40 Airoldi I, Di Carlo E, Cocco C, et al. Lack of IL12RB2 signaling predisposes to spontaneous autoimmunity and malignancy. Blood 2005;106:3846-53

41 Zhao B, Meng L-Q, Huang $\mathrm{H}-\mathrm{N}$, et al. A novel functional polymorphism, $16974 \mathrm{~A} / \mathrm{C}$, in the interleukin-12-3' untranslated region is associated with risk of glioma. DNA Cell Biol 2009;28:335-41.

42 Granier C, De Guillebon E, Blanc C, et al. Mechanisms of action and rationale for the use of checkpoint inhibitors in cancer. ESMO Open 2017;2:e000213.

43 Ngiow SF, Young A, Blake SJ, et al. Agonistic CD40 mAb-Driven IL12 reverses resistance to anti-PD1 in a T-cell-Rich tumor. Cancer Res 2016;76:6266-77.

44 Garris CS, Arlauckas SP, Kohler RH, et al. Successful anti-PD-1 cancer immunotherapy requires T Cell-Dendritic cell crosstalk involving the cytokines IFN- $\gamma$ and IL-12. Immunity 2018;49:1148-61.

45 Barderas R, Bartolomé RA, Fernandez-Aceñero MJ, et al. High expression of IL-13 receptor $\alpha 2$ in colorectal cancer is associated with invasion, liver metastasis, and poor prognosis. Cancer Res 2012;72:2780-90.

46 Suzuki A, Leland P, Joshi BH, et al. Targeting of IL-4 and IL-13 receptors for cancer therapy. Cytokine 2015;75:79-88.

$47 \mathrm{Cao} \mathrm{H}$, Zhang J, Liu H, et al. II-13/Stat6 signaling plays a critical role in the epithelial-mesenchymal transition of colorectal cancer cells. Oncotarget 2016;7:61183-98.

48 Hallett MA, Venmar KT, Fingleton B. Cytokine stimulation of epithelial cancer cells: the similar and divergent functions of IL-4 and IL-13. Cancer Res 2012;72:6338-43.

49 Venmar KT, Carter KJ, Hwang DG, et al. II4 receptor ILR $4 \alpha$ regulates metastatic colonization by mammary tumors through multiple signaling pathways. Cancer Res 2014;74:4329-40. 
50 Bhattacharya S, Mathew G, Jayne DG, et al. 15-Lipoxygenase-1 in colorectal cancer: a review. Tumour Biol 2009;30:185-99.

51 Hsi LC, Xi X, Wu Y, et al. The methyltransferase inhibitor 5-aza-2deoxycytidine induces apoptosis via induction of 15-lipoxygenase-1 in colorectal cancer cells. Mol Cancer Ther 2005;4:1740-6.

52 Hsi LC, Xi X, Lotan R, et al. The histone deacetylase inhibitor suberoylanilide hydroxamic acid induces apoptosis via induction of 15-lipoxygenase-1 in colorectal cancer cells. Cancer Res 2004;64:8778-81.

53 Ma Y, Hwang RF, Logsdon CD, et al. Dynamic mast cell-stromal cell interactions promote growth of pancreatic cancer. Cancer Res 2013;73:3927-37.

54 Jiang L, Cheng Q, Zhang B, et al. II-13 induces the expression of $11 \beta \mathrm{HSD} 2$ in IL-13R $\alpha 2$ dependent manner and promotes the malignancy of colorectal cancer. Am J Transl Res 2016;8:1064-72.

55 Dalessandri T, Crawford G, Hayes M, et al. II-13 from intraepithelial lymphocytes regulates tissue homeostasis and protects against carcinogenesis in the skin. Nat Commun 2016;7:12080.

56 Hsi LC, Kundu S, Palomo J, et al. Silencing IL-13R 22 promotes glioblastoma cell death via endogenous signaling. Mol Cancer Ther 2011;10:1149-60.
57 Chang $\mathrm{Y}, \mathrm{Xu} \mathrm{L}, \mathrm{An} \mathrm{H}$, et al. Expression of IL-4 and IL-13 predicts recurrence and survival in localized clear-cell renal cell carcinoma. Int $J$ Clin Exp Pathol 2015;8:1594-603.

58 Zurita AJ, Jonasch E, Wang X, et al. A cytokine and angiogenic factor (CAF) analysis in plasma for selection of sorafenib therapy in patients with metastatic renal cell carcinoma. Ann Oncol 2012;23:46-52.

$59 \mathrm{Xu} \mathrm{W}$, Puligandla M, Manola J, et al. Angiogenic factor and cytokine analysis among patients treated with adjuvant VEGFR TKIs in resected renal cell carcinoma. Clin Cancer Res 2019;25:6098-106.

60 Peña C, Lathia C, Shan M, et al. Biomarkers predicting outcome in patients with advanced renal cell carcinoma: results from sorafenib phase III treatment approaches in renal cancer global evaluation trial. Clin Cancer Res 2010;16:4853-63.

61 Mauge L, Mejean A, Fournier L, et al. Sunitinib prior to planned nephrectomy in metastatic renal cell carcinoma: angiogenesis biomarkers predict clinical outcome in the prospective phase I PREINSUT trial. Clin Cancer Res 2018;24:5534-42.

62 Sun X, Xiang C-J, Wu J, et al. Relationship between serum inflammatory cytokines and lifestyle factors in gastric cancer. Mol Clin Oncol 2019;10:401-14. 